\title{
Androgen Insensitivity Syndrome: Clinical Phenotype and Molecular Analysis in a Single Tertiary Center Cohort
}

\author{
(D) Maria Sol Touzon*1,2, (D) Natalia Perez Garrido*1, (D) Roxana Marino ${ }^{1}$, (D) Pablo Ramirez¹, (D) Mariana Costanzo1, \\ (D) Gabriela Guercio1,2, (D) Esperanza Berensztein1, (D) Marco A. Rivarola1,2, (D) Alicia Belgorosky1,2 \\ ${ }^{1}$ Hospital de Pediatria Garrahan, Endocrinology Service, Buenos Aires, Argentina \\ ${ }^{2}$ National Scientific and Technical Research Council (CONICET), Endocrinology Service, Buenos Aires, Argentina \\ *These two authors contributed equally to this work.
}

\section{What is already known on this topic?}

Androgen insensitivity syndromes (AIS) the most frequent known monogenic cause of 46,XY disorder of sexual differentiation. Mutations of variable severity in androgen receptor gene are associated with a wide phenotypic spectrum, ranging from complete AIS to a partial form or a mild form.

\section{What this study adds?}

Characterization of the clinical phenotype, long term follow up, in particular gender identityand the contribution of the $A R$ gene to the molecular cause of 46,XY disorder of sexual differentiation in a single tertiary pediatric center of Buenos Aires, Argentina are reported. Nine novel $A R$ mutations are described

\section{Abstract}

Objective: The aim of this study was the molecular characterization of the $A R$ gene as the cause of 46,XY disorder in our population. Methods: We studied 41, non related, 46,XY disorder of sexual differentiation index cases, having characteristics consistent with androgen insensivity syndrome (AIS). Genomic DNA was isolated from peripheral blood leukocytes of all patients and 25 family members from 17 non-related families.

Results: The $A R$ gene analysis revealed an abnormal sequence in $58.5 \%$ of the index patients. All of the complete AIS (CAIS) cases were genetically confirmed, while in the partial form (PAIS) a mutation in $A R$ was detected in only 13 (43.3\%). Molecular studies revealed other affected or carrier relatives in $87 \%$ of the index cases. The $A R$ mutations were found spread along the whole coding sequence, with a higher prevalence in the ligand binding domain. Nine out of 23 (39\%) AR mutations were novel. In $17 \%$ of patients with detected $A R$ mutations, somatic mosaicism was detected in leucocyte DNA. In our cohort, long-term follow up gender dysphoria, raised as male or female, was not found. Finally, in suspected PAIS, the identification of AR mutation occurred significantly less than in CAIS patients. Conclusion: Improved knowledge of the components of the $A R$ complex and signaling network might contribute to long term outcome and genetic counseling in AIS patients.

Keywords: 46,XY disorders of sex development, androgen insensitivity syndrome, androgen receptor gene mutations, mosaicism, clinical phenotype

\section{Introduction}

The endogenous androgens, testosterone (T) and dihydrotestosterone (DHT), exert their effects via a single intracellular receptor protein, the androgen receptor $(A R)$
(1). AR-mediated androgen action is essential for normal primary male sexual development before birth and for normal secondary male sexual development around puberty, whereas in females, androgens also participate in sexual development around puberty and in adult
Address for Correspondence: Alicia Belgorosky MD, Hospital de Pediatria Garrahan, Endocrinology Service, Buenos Aires, Argentina Phone: +541143080034 E-mail: abelgo12345@gmail.com ORCID ID: orcid.org/0000-0002-4234-400X

'Copyright 2019 by Turkish Pediatric Endocrinology and Diabetes Society

The Journal of Clinical Research in Pediatric Endocrinology published by Galenos Publishing House.
Conflict of interest: None declared Received: 24.07.2018 Accepted: 20.09 .2018 
female sexual function (2). The $A R$ gene is located on the $\mathrm{X}$-chromosome inthe $\mathrm{Xq} 11-12$ region and encodes a protein with a molecular mass of approximately $110 \mathrm{kDa}$. The gene consists of eight coding exons (I to VIII) (3). The $A R$ is a transcription factor that belongs to the nuclear receptor subfamily 3 , group $C$, member 4 . The protein consists of 920 amino acids that, like other nuclear receptors, is composed of an N-terminal domain (NTD), located on exon 1, a DNA-binding domain (DBD), located on exons 2 and 3 containing two zinc fingers, a hinge region connecting the ligand-binding domain (LBD) to the DBD and a C-terminal LBD, located on exons 4-8 (4).

Androgen insensitivity syndrome (AIS; OMIM 300068) is the most frequent known monogenic cause of $46, \mathrm{XY}$ disorders of sex development (DSD) and is an X-linked recessive condition. Mutations of variable severity in the $A R$ gene are associated with a wide phenotypic spectrum, ranging from complete AIS (CAIS) to a partial form (PAIS) or a mild form (MAIS). Patients who present with CAIS exhibit female external genitalia, testes located in the inguinal or abdominal area, and complete breast development with sparse to absent axillary and pubic hair. Patients with PAIS present with a predominantly male phenotype with hypospadias or a predominantly female phenotype with cliteromegaly and/or posterior labial fusion, ambiguous genitalia and variable degrees of gynecomastia at puberty. Patients with MAIS present with normal external male genitalia associated with infertility (5).

The aim of this study was to characterize the clinical phenotype and the contribution of the $A R$ gene to the molecular cause of 46,XY DSD in our population.

\section{Methods}

We studied 41 unrelated 46,XY DSD patients with clinical and hormonal characteristics consistent with AIS. CAIS was suspected in 11 of the patients and PAIS in 30. All patients presented with female or ambiguous external genitalia, adequate $\mathrm{T}$ production without evidence of steroidogenic blockade and no Müllerian structures evident on abdominal ultrasound. Patients with hormonal determinations previous to gonadal biopsy or gonadectomy presented no biochemical evidence of gonadal dysgenesis and had normal male follicle-stimulating hormone (FSH) levels. In these individuals, the $A R$ gene was the first candidate for molecular analysis.

Informed consent for the genetic study was obtained from all of the patients or their parents guardians after full explanation of the purpose and nature of all procedures.
The study was approved by the Independent Ethics Committee "Prof. Dr. J. P. Garrahan Pediatric Hospital" (reference number: 971).

\section{Hormonal Assays}

Serum luteinising hormone (LH) and FSH levels were determined by the IMX systems (Abbott Laboratories, Abbott Park, IL); assay sensitivity was $0.3 \mathrm{IU} / \mathrm{L}$ for $\mathrm{LH}$ and $0.2 \mathrm{IU} / \mathrm{L}$ for $\mathrm{FSH}$; interassay coefficient of variation ranged from 3.1-8.7\% for LH and from 3.8-12\% for FSH. Serum anti-Müllerian hormone (AMH) levels were determined by ELISA; assay sensitivity was $0.5 \mathrm{pmol} / \mathrm{L}$ and assay limit of quantification $1.2 \mathrm{pmol} / \mathrm{L}$ serum $\mathrm{T}$ was determined by a DPC Immulite ${ }^{\circledR}$ Assay System (Diagnostic Products, Los Angeles, CA); assay sensitivity was $0.17 \mathrm{nmol} / \mathrm{L}$; interassay coefficient of variation ranged from 7.4 to $13 \%$.

\section{$A R$ Gene Mutation Analysis}

Genomic DNA was isolated from peripheral blood leukocytes of all patients (41 index cases) and 25 family members from 17 families according to standard procedures. There were seven families in which family members were not available for molecular studies. The entire coding region (exons 1-8) and splice sites in flanking intronic regions of $A R$ gene were polymerase chain reaction (PCR) amplified and sequenced by automated analysers (6).

After PCR, the products were assessed by electrophoresis on a $1 \%$ agarose gel stained with ethidium bromide and showed a single band with expected size. The PCR products were purified (Qia Quick PCR Purification Kit, Qiagen, Buenos Aires, Argentina) and sequenced using a BigDye Terminator version 3.1 cycle sequencing kit (Applied Biosystems, Buenos Aires, Argentina) on an ABI PRISM ${ }^{\circledR}$ 3130 Genetic Analyzer capillary DNA sequencer (Applied Biosystems, Buenos Aires, Argentina). The primers used for sequencing were the same as those used for PCR. Previously reported intronic mutations were also analysed [Human Gene Mutation Database (HGMD), www.hgmd.cf.ac.uk/]. The nucleotide sequences obtained were compared with those from Genebank accession number: NG_009014.2. Nucleotide changes were reconfirmed in each sample DNA by antisense sequence and resequencing after a new PCR product was produced from the original DNA extract.

\section{In silico Protein Analysis}

Nonsense and frameshift mutations which implicate a premature stop codon and a truncated protein were considered deleterious.

The sequence homology-based tool, [Sorting Intolerant from Tolerant (SIFT); http://sift.jcvi.org/], version 2.0.6, 
the structure-based tool PolyPhen-2 (Polymorphism Phenotyping v2, http://genetics.bwh.harvard.edu/pph2/) and Mutation Taster (http://www.mutationtaster.org/) were used to predict the pathogenicity of the previously undescribed missense variants using default settings. To evaluate the implication of a novel synonymous mutation, we used The Berkeley Drosophila Genome Project (http:// www.fruitfly.org/) as a splice site prediction program.

The SIFT algorithm predicts the functional importance of the substitutions based on the alignment of orthologous and/or paralogous protein sequences. The PolyPhen-2 algorithm predicts the functional effects of amino acid changes by considering conservation, physicochemical differences and the proximity of the substitution to the predicted functional domains. Unlike SIFT or PolyPhen which handle only single amino acid substitutions, MutationTaster works on the DNA level and allows insertions and deletions up to 12 base pairs.
The original sequence of the protein was obtained from the Ensembl and UniProt/Swiss-Prot databases.

\section{Statistical Analysis}

This study describes the genotype and clinical phenotype of patients with AIS. A statistical analysis was not necessary.

\section{Results}

In our study $A R$ gene analysis revealed an abnormal sequence in 24 individuals (58.5\% of total index patients). All of the CAIS cases $(n=11)$ were genetically confirmed, while in PAIS $(\mathrm{n}=30)$ a mutation in $A R$ was detected in only 13 patients $(43.3 \%)$.

Family studies were performed in 25 family members from 17 families. The molecular studies and affected family members are shown in Table 1 . Molecular studies revealed other affected or carrier relatives in $87 \%$ of the index cases.

Table 1. Clinical phenotype, social sex and molecular studies

\begin{tabular}{|c|c|c|c|c|c|c|c|}
\hline \multirow[b]{2}{*}{ Patient } & \multirow[b]{2}{*}{ Clinical form } & \multirow{2}{*}{$\begin{array}{l}\text { Social } \\
\text { sex }\end{array}$} & \multirow[b]{2}{*}{ Mutation } & \multicolumn{2}{|c|}{ Location } & \multirow[b]{2}{*}{ Type of AIS } & \multirow[b]{2}{*}{ Family studies } \\
\hline & & & & $\begin{array}{l}\text { Protein } \\
\text { domain }\end{array}$ & Exon & & \\
\hline 1 & CAIS & $\mathrm{F}$ & $\begin{array}{l}\text { ap.Trp399Valfs } * 95 \\
\text { c. } 1197 \_1213 \text { del }\end{array}$ & NTD & 1 & Hereditary & Mother/Sistercarriers \\
\hline 2 & CAIS & $\mathrm{F}$ & $\begin{array}{l}\text { p.Leu822Pro } \\
\text { c. } 2464 \mathrm{~T}>\mathrm{C} \\
(27)\end{array}$ & LBD & 7 & Hereditary & Mother/Sistercarriers \\
\hline 3 & CAIS & $\mathrm{F}$ & $\begin{array}{l}\text { p.Arg832* } \\
\text { c. } 2494 \mathrm{C}>\mathrm{T} \\
(28)\end{array}$ & LBD & 7 & De novo & Notdetected (mother) \\
\hline 4 & CAIS & $\mathrm{F}$ & $\begin{array}{l}\text { p.Pro767Ser } \\
\text { c. } 2299 \text { C > T } \\
(26)\end{array}$ & LBD & 5 & Hereditary & Mothercarrier \\
\hline 5 & PAIS & M & $\begin{array}{l}\text { p. }[\text { Cys602 = /Cys602Phe }] \\
\text { c. }[=/ 1805 \mathrm{G}>\mathrm{T}] \\
(29)\end{array}$ & DBD & 3 & $\begin{array}{l}\text { De novo, } \\
\text { mosaic }\end{array}$ & Not detected (mother) \\
\hline 6 & PAIS & $\mathrm{F}$ & $\begin{array}{l}\text { ap. }[\text { Glu } 804=/ \text { Glu } 804 *] \\
\text { c. }[=/ 2410 G>T]\end{array}$ & LBD & 6 & $\begin{array}{l}\text { De novo, } \\
\text { mosaic }\end{array}$ & Not detected (mother) \\
\hline 7 & CAIS & $\mathrm{F}$ & $\begin{array}{l}\text { p.Ile899Phe } \\
\text { c. } 2695 \mathrm{~A}>\mathrm{T} \\
(30)\end{array}$ & LBD & 8 & - & Not done \\
\hline 8 & PAIS & $\mathrm{F}$ & $\begin{array}{l}\text { ap. }[\text { His730 = /His730Glnfs*38] } \\
\text { c. }\left[=/ 2188 \_2194 \text { dupACTTACA }\right]\end{array}$ & LBD & 5 & Mosaic & Not done \\
\hline 9 & CAIS & $\mathrm{F}$ & $\begin{array}{l}\text { p.Met750Val } \\
\text { c. } 2248 \mathrm{~A}>\mathrm{G} \\
(31)\end{array}$ & LBD & 5 & - & Not done \\
\hline 10 & PAIS & M & $\begin{array}{l}\text { p.Arg608Gln } \\
\text { c. } 823 \mathrm{G}>\mathrm{A} \\
(32)\end{array}$ & DBD & 3 & Hereditary & $\begin{array}{l}\text { Mother/aunt/ } \\
\text { Grandmother carriers } \\
\text { Cousin affected }\end{array}$ \\
\hline
\end{tabular}




\begin{tabular}{|c|c|c|c|c|c|c|c|}
\hline \multirow[b]{2}{*}{ Patient } & \multirow[b]{2}{*}{ Clinical form } & \multirow[b]{2}{*}{$\begin{array}{l}\text { Social } \\
\text { sex }\end{array}$} & \multirow[b]{2}{*}{ Mutation } & \multicolumn{2}{|c|}{ Location } & \multirow[b]{2}{*}{ Type of AIS } & \multirow[b]{2}{*}{ Family studies } \\
\hline & & & & $\begin{array}{l}\text { Protein } \\
\text { domain }\end{array}$ & Exon & & \\
\hline 11 & CAIS & $\mathrm{F}$ & $\begin{array}{l}\text { p. Val890Met } \\
\text { c. } 2668 G>A \\
(33)\end{array}$ & LBD & 8 & Hereditary & Mother carrier \\
\hline 12 & PAIS & $\mathrm{F}$ & $\begin{array}{l}\text { p.Asp691 del } \\
\text { c.2071_2073del } \\
(34)\end{array}$ & LBD & 4 & Hereditary & $\begin{array}{l}\text { Mother carrier/ } \\
\text { Sister affected }\end{array}$ \\
\hline 13 & CAIS & $\mathrm{F}$ & $\begin{array}{l}\text { ap.Phe726Cys } \\
\text { c. } 2176 \mathrm{~T}>\mathrm{G}\end{array}$ & LBD & 5 & Hereditary & Mother carrier \\
\hline 14 & CAIS & $\mathrm{F}$ & $\begin{array}{l}\text { p.Arg856Cys } \\
\text { c. } 2566 C>\mathrm{T} \\
(35)\end{array}$ & LBD & 7 & - & Not done \\
\hline 15 & CAIS & $\mathrm{F}$ & $\begin{array}{l}\text { ap.Gln658Argfs*3 } \\
\text { c.1972_1973del }\end{array}$ & LBD & 4 & Hereditary & $\begin{array}{l}1 \text { Sister affected/ } \\
1 \text { Sister carrier }\end{array}$ \\
\hline 16 & PAIS & M & $\begin{array}{l}\text { ap. }\left[\mathrm{Gln} 98=/ \mathrm{Gln} 98 \mathrm{Hisfs}^{*} 8\right] \\
\text { c. }\left[=/ 292 \_311 \mathrm{del}\right]\end{array}$ & NTD & 1 & Mosaic & Not done \\
\hline 17 & CAIS & $\mathrm{F}$ & $\begin{array}{l}\text { ex2-ex8del } \\
\text { C.1617-1768_2608-2763del } \\
\text { (36) }\end{array}$ & $\begin{array}{l}\text { DBD- } \\
\text { LBD }\end{array}$ & $2-8$ & - & Not done \\
\hline 18 & PAIS & M & $\begin{array}{l}\text { p.Arg841 Cys } \\
\text { c. } 2521 \mathrm{C}>\mathrm{T} \\
(35)\end{array}$ & LBD & 7 & Hereditary & Mother carrier \\
\hline 19 & PAIS & M & $\begin{array}{l}\text { p.Arg841 Cys } \\
\text { c. } 2521 \mathrm{C}>\mathrm{T} \\
(35)\end{array}$ & LBD & 7 & Hereditary & Mother carrier \\
\hline 20 & PAIS & M & $\begin{array}{l}\text { p. }(\operatorname{Ser} 889=) \\
\text { c. } 2667 \mathrm{C}>\mathrm{T} \\
(37)\end{array}$ & LBD & 8 & - & Not done \\
\hline 21 & PAIS & M & $\begin{array}{l}\text { p.Ala597Thr } \\
\text { c. } 1789 \mathrm{G}>\mathrm{A} \\
(38)\end{array}$ & DBD & 3 & Hereditary & Mother carrier \\
\hline 22 & PAIS & M & $\begin{array}{l}\text { ap.Asn611 Ile } \\
\text { c. } 1832 \mathrm{~A}>\mathrm{T}\end{array}$ & DBD & 3 & Hereditary & Mother carrier \\
\hline 23 & PAIS & M & $\begin{array}{l}\text { ap.Glu707Asp } \\
\text { c. } 2121 \text { A > C }\end{array}$ & LBD & 4 & Hereditary & Mother carrier \\
\hline 24 & PAIS & M & $\begin{array}{l}\text { ap.His886Tyr } \\
\text { c. } 2656 \mathrm{C}>\mathrm{T}\end{array}$ & LBD & 8 & Hereditary & Mother carrier \\
\hline
\end{tabular}

anovel mutation. CAIS: complete androgen insensitivity syndrome, PAIS: partial androgen insensitivity syndrome, F: female, M: male, NTD: N-terminal domain, DBD: DNA-binding domain, LBD: ligand-binding domain, AIS: androgen insensitivity syndromes

De novo AR mutations were found in three (P3, P5 and P6) out of 13 mothers analyzed. In two non-related index cases (P12 A and P15), two 46,XY affected siblings raised as female were detected. Interestingly, even though in P12A PAIS was established, normal external female genitalia, in the affected sister, was observed (P12 B). As shown in Table 1, $23 A R$ mutations were detected. The $A R$ mutations were found spread along the whole coding sequence, with a higher prevalence in LBD: $8.3 \%$ were located in NTD; $16.6 \%$ in the DBD; $70.8 \%$ in the LBD and $4.3 \%$ were gross deletions (7).

Nine out of 23 (39\%; P1, P6, P8, P13, P15, P16, P22, P23 and P24) AR mutations were novel. Two novel mutations were located in the NTD domain (P1 and P16). They 
were both out of frame deletions that ultimately created a nonsense stop codon and premature truncation of the protein. The others, located in the LBD, were:four missense mutations, a nonsense mutation together with a $2 \mathrm{bp}$ deletion and a duplication of $7 \mathrm{bp}$ that produce a frameshift with a premature stop codon. Three patients (P6, P8 and P16) harboured somatic mosaicisms: a nonsense mutation, a $7 \mathrm{bp}$ duplication and a $20 \mathrm{bp}$ deletion which result in a truncating frameshift mutation. One missense mutation was located in the DBD. All novel mutations were predicted to be pernicious by all in silico tools.
In four individuals (P5, P6, P8 and P16), $17 \%$ of AR-mutated gene patients, somatic mosaicism of mutant and wild type alleles was detected in DNA derived from blood leukocytes.

Of the 17 individuals without a defect in the AR, two patients were finally diagnosed (and genetically confirmed) with 5-alpha reductase deficiency. In the others, diagnosis remains unknown.

The clinical phenotype and follow-up of the genetically confirmed patients is shown in Supplemental Table 1. Interestingly, during follow-up, no gender dysphoria,

\section{Supplemental Table 1. Clinical phenotype at diagnosis and long term follow-up}

\begin{tabular}{|c|c|c|c|c|c|c|c|c|}
\hline Patient & $\begin{array}{l}\text { CA at } \\
\text { diagnosis } \\
\text { (years) }\end{array}$ & $\begin{array}{l}\text { Clinical } \\
\text { form }\end{array}$ & Phenotype & $\begin{array}{l}\text { Gonadal } \\
\text { position }\end{array}$ & $\begin{array}{l}\text { EMS at } \\
\text { diagnosis } \\
\text { (PAIS) }\end{array}$ & $\begin{array}{l}\text { Social } \\
\text { sex }\end{array}$ & $\begin{array}{l}\text { Follow-up: } \\
\text { puberty, } \\
\text { gender } \\
\text { dysphoria }\end{array}$ & $\begin{array}{l}\text { Hormonal } \\
\text { profile }\end{array}$ \\
\hline 1 & 4 & CAIS & $\begin{array}{l}\text { Female genitalia, } \\
\text { inguinal hernia }\end{array}$ & Inguinal & & $\mathrm{F}$ & $\begin{array}{l}\text { Female gender } \\
\text { identity. }\end{array}$ & \\
\hline 2 & 5.7 & CAIS & $\begin{array}{l}\text { Female genitalia, } \\
\text { inguinal hernia }\end{array}$ & Inguinal & & $\mathrm{F}$ & $\begin{array}{l}\text { Lost to follow- } \\
\text { up. }\end{array}$ & \\
\hline 3 & & CAIS & $\begin{array}{l}\text { Female genitalia, } \\
\text { inguinal hernia }\end{array}$ & Inguinal & & $\mathrm{F}$ & & \\
\hline 4 & 6.7 & CAIS & $\begin{array}{l}\text { Female genitalia, } \\
\text { inguinal hernia }\end{array}$ & Inguinal & & $\mathrm{F}$ & $\begin{array}{l}\text { Female gender } \\
\text { identity. }\end{array}$ & \\
\hline 5 & 1.7 & PAIS & $\begin{array}{l}\text { Penoscrotal } \\
\text { hypospadias }\end{array}$ & Scrotal & 9 & M & $\begin{array}{l}\text { Pubertal onset } \\
\text { at } 9.6 \text { years, } \\
\text { gynecomastia, } \\
\text { male gender } \\
\text { identity. }\end{array}$ & $\begin{array}{l}\text { Gonadotropins, } \\
\mathrm{T} \text { and } \mathrm{AMH} \\
\text { levels according } \\
\text { to male } \\
\text { reference range. }\end{array}$ \\
\hline 6 & 0.5 months & PAIS & $\begin{array}{l}\text { Micropenis, } \\
\text { penoscrotal } \\
\text { hypospadias }\end{array}$ & Scrotal & 6 & M & $\begin{array}{l}\text { Spontaneous } \\
\text { pubertal onset, } \\
\text { pubertal } \\
\text { development } \\
\text { not available. } \\
\text { Gender identity } \\
\text { not available. }\end{array}$ & \\
\hline 7 & 0.3 months & PAIS & $\begin{array}{l}\text { Micropenis, } \\
\text { penoscrotal } \\
\text { hypospadias }\end{array}$ & Inguinal/scrotal & 5.5 & $\mathrm{~F}$ & $\begin{array}{l}\text { Female gender } \\
\text { identity. Speech } \\
\text { delay. }\end{array}$ & $\begin{array}{l}\text { Gonadotropins, } \\
\mathrm{T} \text {, and AMH } \\
\text { levels according } \\
\text { to male } \\
\text { reference range } \\
\text { in the neonatal } \\
\text { period. }\end{array}$ \\
\hline 8 & 0.8 months & PAIS & $\begin{array}{l}\text { Micropenis, } \\
\text { penoscrotal } \\
\text { hypospadias }\end{array}$ & Scrotal & 6 & M & $\begin{array}{l}\text { Lost to follow- } \\
\text { up }\end{array}$ & \\
\hline 9 & 9.8 & PAIS & $\begin{array}{l}\text { Female genitalia, } \\
\text { clitoris hypertrophy }\end{array}$ & NA & NA & $\mathrm{F}$ & $\begin{array}{l}\text { Female gender } \\
\text { identity, } \\
\text { hypoplastic } \\
\text { upper vagina } \\
\text { requiring } \\
\text { sigmoidal } \\
\text { vaginal } \\
\text { replacement. }\end{array}$ & $\begin{array}{l}\text { Gonadotropins, } \\
\mathrm{T} \text {, and AMH } \\
\text { levels according } \\
\text { to male } \\
\text { reference range } \\
\text { in the neonatal } \\
\text { period. }\end{array}$ \\
\hline 10 & 4 months & CAIS & $\begin{array}{l}\text { Female genitalia, } \\
\text { inguinal hernia }\end{array}$ & Inguinal & & F & $\begin{array}{l}\text { Female gender } \\
\text { identity. }\end{array}$ & \\
\hline
\end{tabular}


Supplemental Table 1. Continued

\begin{tabular}{|c|c|c|c|c|c|c|c|c|}
\hline 11 & 9.8 & CAIS & $\begin{array}{l}\text { Female } \\
\text { genitalia, } \\
\text { inguinal hernia }\end{array}$ & Inguinal & & $\mathrm{F}$ & $\begin{array}{l}\text { Female gender identity. } \\
\text { Wolf-Parkinson White } \\
\text { syndrome. }\end{array}$ & $\begin{array}{l}\text { Gonadotropins } \\
\text { levels according } \\
\text { to male reference } \\
\text { range. }\end{array}$ \\
\hline 12 & $\begin{array}{l}1.5 \\
\text { months }\end{array}$ & PAIS & $\begin{array}{l}\text { Penoscrotal } \\
\text { hypospadias }\end{array}$ & Scrotal & 9 & M & $\begin{array}{l}\text { Precocious pubertal } \\
\text { development (onset } \\
\text { at } 7 \text { years), mild } \\
\text { gynecomastia, male } \\
\text { gender identity. }\end{array}$ & $\begin{array}{l}\text { Gonadotropins, T } \\
\text { and AMH levels } \\
\text { in according male } \\
\text { reference range. }\end{array}$ \\
\hline 13 & 2 months & CAIS & $\begin{array}{l}\text { Female } \\
\text { genitalia, } \\
\text { inguinal hernia }\end{array}$ & Inguinal & & $\mathrm{F}$ & Female gender identity. & $\begin{array}{l}\text { Gonadotropins, T } \\
\text { and AMH levels } \\
\text { according to } \\
\text { male neonatal } \\
\text { reference range. }\end{array}$ \\
\hline 14 & 7.3 & PAIS & $\begin{array}{l}\text { Female } \\
\text { genitalia, } \\
\text { complete } \\
\text { fusion of the } \\
\text { labia majora }\end{array}$ & Inguinal & 5 & $\mathrm{~F}$ & Female gender identity. & $\begin{array}{l}\text { Gonadotropins, T } \\
\text { and AMH levels } \\
\text { according to male } \\
\text { reference range. }\end{array}$ \\
\hline 15 & 1.8 & PAIS & $\begin{array}{l}\text { Female } \\
\text { genitalia, } \\
\text { posterior } \\
\text { fusion of the } \\
\text { labia majora, } \\
\text { inguinal hernia }\end{array}$ & Inguinal & 2 & $\mathrm{~F}$ & $\begin{array}{l}\text { Female gender identity. } \\
\text { Obesity. }\end{array}$ & $\begin{array}{l}\text { Gonadotropins } \\
\text { and T levels } \\
\text { according to male } \\
\text { reference range. }\end{array}$ \\
\hline 17 & 7 & CAIS & $\begin{array}{l}\text { Female } \\
\text { genitalia, } \\
\text { inguinal hernia }\end{array}$ & Inguinal & & $\mathrm{F}$ & $\begin{array}{l}\text { Peripheral precocious } \\
\text { puberty secondary to } \\
\text { unilateral gonadal tumor } \\
\text { (Sertoli cell + sexual } \\
\text { cords) }\end{array}$ & \\
\hline 18 & 1.3 & PAIS & $\begin{array}{l}\text { Micropenis, } \\
\text { penoscrotal } \\
\text { hypospadias }\end{array}$ & Scrotal & 6 & M & $\begin{array}{l}\text { Spontaneous pubertal } \\
\text { development, severe } \\
\text { gynecomastia. Gender } \\
\text { identity not available. }\end{array}$ & $\begin{array}{l}\text { Gonadotropins } \\
\text { and T levels } \\
\text { according to male } \\
\text { reference range. }\end{array}$ \\
\hline 19 & 17 & CAIS & $\begin{array}{l}\text { Female } \\
\text { genitalia, } \\
\text { inguinal hernia }\end{array}$ & Inguinal & & $\mathrm{F}$ & $\begin{array}{l}\text { Spontaneous pubertal } \\
\text { development, } \\
\text { female gender } \\
\text { identity. Postpubertal } \\
\text { gonadectomy. }\end{array}$ & $\begin{array}{l}\text { Gonadotropins } \\
\text { and T levels } \\
\text { according to male } \\
\text { reference range. }\end{array}$ \\
\hline 20 & $\begin{array}{l}0.7 \\
\text { months }\end{array}$ & PAIS & $\begin{array}{l}\text { Penoscrotal } \\
\text { hypospadias }\end{array}$ & Scrotal & 9 & M & $\begin{array}{l}\text { Spontaneous pubertal } \\
\text { development, severe } \\
\text { gynecomastia, male } \\
\text { gender identity. }\end{array}$ & $\begin{array}{l}\text { Increased } \\
\text { neonatal } \\
\text { gonadotropins, } \\
\text { and T levels for } \\
\text { male reference } \\
\text { range. }\end{array}$ \\
\hline
\end{tabular}




\begin{tabular}{|c|c|c|c|c|c|c|c|c|}
\hline \multicolumn{9}{|c|}{ Supplemental Table 1. Continued } \\
\hline Patient & $\begin{array}{l}\text { CA at } \\
\text { diagnosis } \\
\text { (years) }\end{array}$ & $\begin{array}{l}\text { Clinical } \\
\text { form }\end{array}$ & Phenotype & $\begin{array}{l}\text { Gonadal } \\
\text { position }\end{array}$ & $\begin{array}{l}\text { EMS at } \\
\text { diagnosis } \\
\text { (PAIS) }\end{array}$ & $\begin{array}{l}\text { Social } \\
\text { sex }\end{array}$ & $\begin{array}{l}\text { Follow-up: } \\
\text { puberty, } \\
\text { gender } \\
\text { dysphoria }\end{array}$ & $\begin{array}{l}\text { Hormonal } \\
\text { profile }\end{array}$ \\
\hline 21 & 7 months & PAIS & $\begin{array}{l}\text { Micropenis, } \\
\text { penoscrotal } \\
\text { hypospadias }\end{array}$ & Scrotal & 6 & M & $\begin{array}{l}\text { Gender identity } \\
\text { and follow- } \\
\text { up not yet } \\
\text { evaluatable } \\
\text { (toddler). }\end{array}$ & $\begin{array}{l}\text { Gonadotropins T } \\
\text { and AMH levels } \\
\text { according to } \\
\text { male reference } \\
\text { range. }\end{array}$ \\
\hline 22 & 0.5 months & PAIS & $\begin{array}{l}\text { Micropenis, } \\
\text { penoscrotal } \\
\text { hypospadias, inguinal } \\
\text { hernia }\end{array}$ & Inguinal & 5 & M & $\begin{array}{l}\text { Gender identity } \\
\text { and follow- } \\
\text { up not yet } \\
\text { evaluatable } \\
\text { (toddler). }\end{array}$ & $\begin{array}{l}\text { Gonadotropins T } \\
\text { and AMH levels } \\
\text { according to } \\
\text { male reference } \\
\text { range. }\end{array}$ \\
\hline 23 & 2 months & PAIS & $\begin{array}{l}\text { Micropenis, } \\
\text { penoscrotal } \\
\text { hypospadias }\end{array}$ & Scrotal & 6 & M & $\begin{array}{l}\text { Gender identity } \\
\text { and follow- } \\
\text { up not yet } \\
\text { evaluatable } \\
\text { (toddler). }\end{array}$ & $\begin{array}{l}\text { Gonadotropins T } \\
\text { and AMH levels } \\
\text { according to } \\
\text { male reference } \\
\text { range. }\end{array}$ \\
\hline 24 & 0.3 months & PAIS & $\begin{array}{l}\text { Penoscrotal } \\
\text { hypospadias, } \\
\text { unilateral } \\
\text { cryptorchidism }\end{array}$ & Inguinal/scrotal & 8.5 & M & $\begin{array}{l}\text { Gender identity } \\
\text { and follow- } \\
\text { up not yet } \\
\text { evaluatable } \\
\text { (toddler). }\end{array}$ & $\begin{array}{l}\text { Gonadotropins } \\
\text { and T levels } \\
\text { according to } \\
\text { male reference } \\
\text { range. Increased } \\
\text { AMH levels. }\end{array}$ \\
\hline
\end{tabular}

CA: chronologycal age, EMS: external masculinization score (39), CAIS: complete androgen insensitivity syndrome, PAIS: partial androgen insensitivity syndrome, F: female, M: male, NA: not available, BMT: bone marrow transplant, T: testosterone, AMH: anti-Müllerian hormone.

Male reference gonadotropin levels (MU/mL). 0-3 months: follicle-stimulating hormone (FSH): $2.43 \pm 1.67$, luteinising hormone (LH): $2.52 \pm 1.74 ; 3-12$ months: FSH: $1.35 \pm 0.81$, LH: $1.21 \pm 1.65 ; 12-24$ months: FSH: $0.90 \pm 0.59$, LH: $0.15 \pm 0.17$; > 24 months: FSH: $1.10 \pm 0.82$, LH: 0.13 \pm 0.32 ; $9-12$ years: FSH: $2.26 \pm 0.96(\mathrm{MU} / \mathrm{mL}), \mathrm{LH}: 0.78 \pm 0.99$.

Male reference testosterone levels $(\mathrm{ng} / \mathrm{mL})$ : $1-5$ months $<0.05-1.77 ; 6-11$ months $\leq 0.07 ; 1-5$ years $\leq 0.25 ; 6-9$ years $\leq 0.30 ; 10-11$ years $0.05-0.50 ; 12-14$ years 0.10-5.72; $15-17$ years 2.20-8.00.

Male reference AMH levels (pmol/L): 0-14 days: 250-1000; 15 days-3 years 400-2400; > 3 years prepubertal Tanner 1 300-1400; >3 years pubertal Tanner 2 701000; > 3 years pubertal Tanner 3 30-400; > 3 years pubertal Tanner 4/5 30-180.

including those PAIS patients assigned male or female sex, were observed. Unfortunately, in toddler patients, gender identity could not be evaluated. According to previous reports, very low frequency of gonadal tumors was found. Only in P17 was a Sertoli cell tumor detected (8).

\section{Discussion}

We describe a series of unrelated patients affected by different degrees of AIS. AR gene mutations are the main cause of $46, \mathrm{XY}$ DSD. To date, the $A R$ gene mutations database (http://www.mcgill.ca/androgendb/) has reported more than 800 different $A R$ mutations from patients with AIS.

In all CAIS cases, $A R$ mutations responsible for the phenotype were identified. However, similar to other cohorts, in PAIS phenotype cases, AR mutations were identified in only $38 \%$. Overall, in our series of 41 index patients, the $A R$ gene proved to be abnormal in $58.5 \%$, confirming the diagnosis. Similarly, Boehmer et al (9) and Audi et al (2) report a frequency of detection of 44$65 \%$ which is in line with our results. In contrast de Silva et al (10) and Akcay et al (11) describe cohorts with 15$18 \%$ of genetically confirmed AIS. In these studies, the significantly lower percentage of $A R$ mutation detection could be due to the presence of overlaps in the clinical presentation of the patients, such as $5-\alpha$ reductase deficiency or the fact that patients with a $\mathrm{T}$ biosynthetic defect were also included. Therefore, it has been proposed that even though AR is essential for virilization, other components of the AR complex and signaling network are required for complete masculinization. It has been suggested that in non-detected cases androgen resistance might be secondary to mutations in the 5'UTR, or other regulatory regions. Moreover, several necessary AR cofactor(s) should also be taken into consideration. Several cofactors, such as coactivators steroid receptor coactivator 1 (SRC1), transcriptional mediators/intermediary factor 2, SRC3 and corepressors nuclear receptor-interacting 
protein 1 , nuclear receptor subfamily 0 group $B$ member 1 , are actively involved in the regulation of $A R$-mediated transcription, and might play an important role in AIS etiopathogenesis $(12,13,14,15)$. Interestingly, in order to confirm androgen resistance, Hornig et al (16) developed a DHT-dependent transcriptional induction of the androgen-regulated $A P O D$ (apolipoprotein D) gene in cultured genital fibroblasts (APOD-assay). However, the usefulness of this $A P O D$ assay needs to be confirmed in a large cohort.

Mutations in the $A R$ gene are distributed throughout the sequence with a preponderance $(70.8 \%)$ located in the LBD (17). In our cohort, nine novel AR mutations were found, expanding the mutational spectrum of $46, \mathrm{XY}$ DSD. In three of these novel mutations, located in the LBD, a truncated, significantly reducedor inactive protein was predicted due to a premature stop codon, secondary to gene deletion (P15), gene duplication (P8) or nonsense mutation (P6). The p.Phe726Cys missense mutation located in the LBD was also detected. Interestingly the study of Quigley et al (18) demonstrated by functional assays that a missense mutation in the same position (p.Phe726Leu),caused the disruption of the N/C terminal interaction of the mutated protein. Hence it might be reasonable to suppose that the novel missense mutation found in our cohort might also affect the transactivation activity of the AR, impairing the binding of the ligand to its LBD. The remaining novel mutations, two gene deletions (P1 and P16) located in the NTD domain, result in a truncated protein due to a premature stop codon.

A lack of correlation between genotype and clinical phenotype has previously been reported (19). Interestingly, in siblings of family 12, harbouring p.Asp691 del mutation, a clinical variability was evident. A CAIS phenotype was observed in one case, while in the other a PAIS phenotype was observed. Petroli et al's (20) study showed, in N/C terminal interaction assays, different profiles of the mutant AR protein in response to DHT stimulation, explaining the phenotypic diversity observed in PAIS cases.

Somatic mosaicism has been reported. Interestingly, even though the patients carried severe AR mutations, PAIS clinical phenotype was reported. In these affected patients the de novo mutation occurred after the zygote stage and probably very early, during the first few cell divisions. Thus, different proportions of cells containing mutant or wild-type protein are present in various tissues of the same individual explaining the mild phenotype. Similarly, in four patients of our cohort (P5, P6, P8 and P16) a severe mutation was detected but presenting with a PAIS phenotype. It is noteworthy that detection of somatic mosaicism in AR has a great impact for patients with AIS because further virilization is possible after birth and this is an important consideration for genetic counseling (21).

No gender dysphoria was observed in our cohort, even though systematic assessment was not available in all cases.

In contrast to previous reports, in this cohort, $\mathrm{AMH}$ serum concentrations during the neonatal period were within the normal male reference range in the only two PAIS cases in whom it was assessed $(22,23)$. $A M H$ gene expression in Sertoli cells is inhibited via the AR receptor pathway (24). The lack of $A R$ expression in Sertoli cells during mini puberty could explain our findings, suggesting that other as yet unidentified factors might be involved in the regulation of AMH synthesis (25).

In agreement with previous reports, normal gonadotropin levels were the most frequent finding (26).

\section{Study Limitations}

Even though all in silico tools predicted the novel mutations to be damaging for protein structure and function, functional assays should be performed to confirm pathogenicity.

\section{Conclusion}

In summary, we report a series of $4146, X Y$ DSD index patients in whom AR was the candidate gene. Molecular diagnosis is useful for genetic counseling of the families. However, similar to other series, the percentage of suspected casesin whom an AR mutation was found wasonly around $60 \%$.

Emerging technological advances might contribute to an increase in the accuracy of determining the etiology in suspected AIS cases.

\section{Acknowledgements}

The authors want to express their thanks to Marta Ciaccio, Monica Warman, Elisa Vaiani, Silvia Gil, Isabel Di Palma, Veronica Forclaz, Maria del Carmen Malosetti, Carmen Riu and Valeria de Dona for their help with the clinical data.

\section{Ethics}

Ethics Committee Approval: The study was approved by the Independent Ethics Committee "Prof. Dr. J. P. Garrahan Pediatric Hospital” (reference number: 971).

Informed Consent: Informed consent for the genetic study was obtained from all of the patients or their parents guardians after full explanation of the purpose and nature of all procedures. 


\section{Peer-review: Externally peer-reviewed.}

\section{Authorship Contributions}

Design: Maria Sol Touzon, Natalia Perez Garrido, Gabriela Guercio, Mariana Costanzo, Roxana Marino, Marco A. Rivarola, Alicia Belgorosky, Data Collection and Analysis: Sol Touzon, Gabriela Guercio, Mariana Costanzo, Roxana Marino, Pablo Ramirez, Esperanza Berensztein, Natalia Perez Garrido, Writing: Maria Sol Touzon, Natalia Perez Garrido.

Financial Disclosure: Supported by grants from the National Scientific and Technical Research Council (CONICET), Argentina and Fondo para la Investigación Científica y Tecnológica (FONCYT), Argentina.

\section{References}

1. GalaniA, Kitsiou-Tzeli S, Sofokleous C, Kanavakis E, Kalpini-Mavrou A. Androgen insensitivity syndrome: clinical features and molecular defects. Hormones (Athens) 2008;7:217-229.

2. Audi L, Fernández-Cancio M, Carrascosa A, Andaluz P, Torán N, Piró C, Vilaró E, Vicens-Calvet E, Gussinyé M, Albisu MA, Yeste D, Clemente M, Hernández de la Calle I, Del Campo M, Vendrell T, Blanco A, MartínezMora J, Granada ML, Salinas I, Forn J, Calaf J, Angerri O, MartinezSopena MJ, Del Valle J, García E, Gracia-Bouthelier R, Lapunzina P, Mayayo E, Labarta JI, Lledó G, Sánchez Del Pozo J, Arroyo J, PérezAytes A, Beneyto M, Segura A, Borrás V, Gabau E, Caimarí M, Rodríguez A, Martínez-Aedo MJ, Carrera M, Castaño L, Andrade M, Bermúdez de la Vega JA; Grupo de Apoyo al Síndrome de Insensibilidad a los Andrógenos (GrApSIA). Novel $(60 \%)$ and recurrent (40\%) androgen receptor gene mutations in a series of 59 patients with a $46, X Y$ disorder of sex development. J Clin Endocrinol Metab 2010;95:18761888. Epub 2010 Feb 11

3. Brinkmann AO. Molecular basis of androgen insensitivity. Mol Cell Endocrinol 2001;179:105-109.

4. Tadokoro-Cuccaro R, Hughes IA. Androgen insensitivity syndrome. Curr Opin Endocrinol Diabetes Obes 2014;21:499-503.

5. Gottlieb B, Trifiro MA. Androgen Insensitivity Syndrome. GeneReviews ${ }^{\circledR}$. University of Washington, Seattle, 1999.

6. Chu J, Zhang R, Zhao Z, Zou W, Han Y, Qi Q, Zhang H, Wang JC, Tao S, Liu X, Luo Z. Male Fertility Is Compatible with an Arg(840) Cys substitution in the AR in a Large Chinese Family affected with Divergent Phenotypes of AR insensitivity Syndrome. J Clin Endocrinol Metab 2002;87:347-351.

7. Gottlieb B, Beitel LK, Nadarajah A, Paliouras M, Trifiro M. The Androgen Receptor Gene Mutations Database: 2012 update. Hum Mutat 2012;33:887-894. Epub 2012 Mar 13

8. Cools M, Wolffenbuttel KP, Hersmus R, Mendonca BB, Kaprová J, Drop SLS, Stoop H, Gillis AJM, Oosterhuis JW, Costa EMF, Domenice S, Nishi MY, WunschL, Quigley CA, T'Sjoen G, Looijenga LHJ. Malignant testicular germ cell tumors in postpubertal individuals with androgen insensitivity: prevalence, pathology and relevance of single nucleotide polymorphism-based susceptibility profiling. Hum Reprod 2017;32:2561-2573.

9. Boehmer AL, Brinkmann O, Brüggenwirth $\mathrm{H}$, van Assendelft $\mathrm{C}$, Otten BJ, Verleun-Mooijman MC, Niermeijer MF, Brunner HG, Rouwé CW, Waelkens JJ, Oostdijk W, Kleijer WJ, van der Kwast TH, de Vroede MA, Drop SL. Genotype versus phenotype in families with androgen insensitivity syndrome. J Clin Endocrinol Metab 2001;86:4151-4160.
10. de Silva KS, Sirisena ND, Wijenayaka HK, Cooray JG, Jayasekara RW, Dissanayake $\mathrm{VH}$. Androgen insensitivity syndrome in a cohort of Sri Lankan children with 46, XY disorders of sex development (46, XY DSD). Ceylon Med J 2015;60:139-142

11. Akcay T, Fernandez-Cancio M, Turan S, Güran T, Audi L, Bereket A. AR and SRD5A2 gene mutations in a series of 51 Turkish 46,XY DSD children with a clinical diagnosis of androgen insensitivity. Andrology 2014;2:572-578. Epub 2014 Apr 16

12. Heemers HV, Tindall DJ. Androgen Receptor (AR) Coregulators: A Diversity of Functions Converging on and Regulating the AR Transcriptional Complex. Endocr Rev 2007;28:778-808. Epub 2007 Oct 16

13. Van de Wijngaart D, Dubbink H, VanRoyen M, Trapman J, Jenster G. Androgen receptor coregulators: Recruitment via the coactivator binding groove. Mol Cell Endocrinol 2012;352:57-69. Epub 2011 Aug 17

14. Känsäkoski J, Jääskeläinen J, Jääskeläinen T, Tommiska J, Saarinen L, Lehtonen R, Hautaniemi S, Frilander MJ, Palvimo JJ, Toppari J, Raivio T. Complete androgen insensitivity syndrome caused by a deep intronic pseudoexon-activating mutation in the androgen receptor gene. Sci Rep 2016;9;6:32819.

15. Hornig NC, de Beaufort C, Denzer F, Cools M, Wabitsch M, Ukat M, Kulle AE, Schweikert HU, Werner R, Hiort O, Audi L, Siebert R, Ammerpohl O, Holterhus PM. A Recurrent Germline Mutation in the 5'UTR of the Androgen Receptor Cause Complete Androgen Insensitivity by Activating Aberrant UORF Translation. PLoS One 2016;11:0154158.

16. Hornig NC, Ukat M, Schweikert HU, Hiort O, Werner R, Drop SL, Cools M, Hughes IA, Audi L, Ahmed SF, Demiri J, Rodens P, Worch L, Wehner G, Kulle AE, Dunstheimer D, Müller-Roßberg E, Reinehr T, Hadidi AT, Eckstein AK, van der Horst C, Seif C, Siebert R, Ammerpohl $\mathrm{O}$, Holterhus PM. Identification of an AR-mutation negative class of androgen insensitivity by determining endogenous AR-activity. J Clin Endocrinol Metab 2016;101:4468-4477. Epub 2016 Sep 1

17. Hughes IA, Werner R, Bunch T, Hiort O. Androgen insensitivity syndrome. Semin Reprod Med 2012;30:432-442. Epub 2012 Oct 8

18. Quigley CA, Tan JA, He B, Zhou ZX, Mebarki F, Morel Y, Forest MG, Chatelain P, Ritzén EM, French FS, Wilson EM. Partial androgen insensitivity with phenotypic variation caused by androgen receptor mutations that disrupt activation function 2 and the $\mathrm{NH}(2)$ - and carboxyl-terminal interaction. Mech Ageing Dev 2004;125:683-695.

19. Williams textbook of Endocrinology 13th ed. Testicular disorders. Philadelphia, PA. Elsevier Saunders, 2016.

20. Petroli RJ, Hiort O, Struve D, Gesing JK, Soardi FC, Spínola-Castro AM, Melo K, Prado Arnhold IJ, Maciel-Guerra AT, Guerra-Junior G, Werner R, de Mello MP. Functional Impact of Novel Androgen Receptor Mutations on the Clinical Manifestation of Androgen Insensitivity Syndrome. Sex Dev 2017;11:238-247. Epub 2017 Dec 14

21. Köhler B, Lumbroso S, Leger J, Audran F, Grau ES, Kurtz F, Pinto G, Salerno M, Semitcheva T, Czernichow P, Sultan C. Androgen insensitivity syndrome: somatic mosaicism of the androgen receptor in seven families and consequences for sex assignment and genetic counseling. J Clin Endocrinol Metab 2005;90:106-111. Epub 2004 Nov 2

22. Rey R, Mebarki F, Forest MG, Mowszowicz I, Cate RL, Morel Y, Chaussain JL, Josso N. Anti-müllerian hormone in children with androgen insensitivity. J Clin Endocrinol Metab 1994;79:960-964.

23. Rey RA, Belville C, Nihoul-Fékété C, Michel-Calemard L, Forest MG, Lahlou N, Jaubert F, Mowszowicz I, David M, Saka N, Bouvattier C, Bertrand AM, Lecointre C, Soskin S, Cabrol S, Crosnier H, Léger J, Lortat-Jacob S, Nicolino M, Rabl W, Toledo SP, Baş F, Gompel A, Czernichow P, Josso N. Evaluation of gonadal function in 107 intersex patients by means of serum antimüllerian hormone measurement. J Clin Endocrinol Metab 1999;84:627-631. 
24. Boukari K, Meduri G, Brailly-Tabard S, Guibourdenche J, Ciampi ML, Massin N, Martinerie L, Picard JY, Rey R, Lombès M, Young J. Lack of androgen receptor expression in Sertoli cells accounts for the absence of anti-Mullerian hormone repression during early human testis development. J Clin Endocrinol Metab 2009;94:181 8-1825. Epub 2009 Mar 10

25. Berensztein EB, Baquedano MS, Gonzalez CR, Saraco NI, Rodriguez J, Ponzio R, Rivarola MA, Belgorosky A. Expression of aromatase, estrogen receptor alpha and beta, androgen receptor, and cytochrome P-450scc in the human early prepubertal testis. Pediatr Res 2006;60:740-744. Epub 2006 Oct 25

26. Ahmed SF, Cheng A, Hughes IA. Assessment of the gonadotrophingonadal axis in androgen insensitivity syndrome. Arch Dis Child 1999;80:324-329.

27. Akella RR. Mutational analysis of androgen receptor gene in two families with androgen insensitivity Indian. Indian J Endocrinol Metab 2017;21:520-523.

28. Audi L, Fernández-Cancio M, Carrascosa A, Andaluz P, Torán N, Piró C, Vilaró E, Vicens-Calvet E, Gussinyé M, Albisu MA, Yeste D, Clemente M, Hernández de la Calle I, Del Campo M, Vendrell T, Blanco A, MartínezMora J, Granada ML, Salinas I, Forn J, Calaf J, Angerri O, MartínezSopena MJ, Del Valle J, García E, Gracia-Bouthelier R, Lapunzina P, Mayayo E, Labarta JI, Lledó G, Sánchez Del Pozo J, Arroyo J, PérezAytes A, Beneyto M, Segura A, Borrás V, Gabau E, Caimarí M, Rodríguez A, Martínez-Aedo MJ, Carrera M, Castaño L, Andrade M, Bermúdez de la Vega JA; Grupo de Apoyo al Síndrome de Insensibilidad a los Andrógenos (GrApSIA). Novel (60\%) and Recurrent (40\%) Androgen Receptor Gene Mutations in a Series of 59 Patientswith a 46,XY Disorder of Sex Development. J Clin Endocrinol Metab 2010;95:18761888. Epub 2010 Feb 11

29. Baldazzi L, Baroncini C, Pirazzoli P, Balsamo A, Capelli M, Marchetti G, Bernardi F, Cacciari E. Two mutations causing complete androgen insensitivity: a frame-shift in the steroid binding domain and a Cys-$>$ Phe substitution in the second zinc finger of the androgen receptor. Hum Mol Genet 1994;3:1169-1170.

30. Melo KF, Mendonca BB, Billerbeck AE, Costa EM, Inácio M, Silva FA, Leal AM, Latronico AC, Arnhold IJ. Clinical, Hormonal, Behavioral, and Genetic Characteristics of Androgen Insensitivity Syndrome in a Brazilian Cohort: Five Novel Mutations in the Androgen Receptor Gene. J Clin Endocrinol Metab 2003;88:3241-3250.

31. Bouvattier C, Carel JC, Lecointre C, David A, Sultan C, Bertrand AM, Morel Y, Chaussain JL. Postnatal changes of T, LH, and FSH in 46, $\mathrm{XY}$ infants with mutations in the AR gene. J Clin Endocrinol Metab 2002;87:29-32.

32. Weidemann W, Linck B, Haupt H, Mentrup B, Romalo G, Stockklauser K, Brinkmann AO, Schweikert HU, Spindler KD. Clinical and biochemical investigations and molecular analysis of subjects with mutations in the androgen receptor gene. Clin Endocrinol (Oxf) 1996;45:733-739.

33. Pinsky L, Trifiro M, Kaufman M, Beitel LK, Mhatre A, Kazemi-Esfarjani P, Sabbaghian N, Lumbroso R, Alvarado C, Vasiliou M. Androgen resistance due to mutation of the androgen receptor. Clin Invest Med 1992; 15:456-472.

34. Schwartz M, Skovby F, Mueller J, Nielsen O, Skakkebaek NE. Partial androgen insensitivity (PAIS) in a large eskimo kindred caused by a del D690 mutation in the androgen receptor (AR) gene. Horm Res 1994;41:117.

35. McPhaul MJ, Marcelli M, Zoppi S, Wilson CM, Griffin JE, Wilson JD. Mutations in the ligand-binding domain of the androgen receptor gene cluster in two regions of the gene. J Clin Invest 1992;90:2097-2101.

36. Jakubiczka S, Nedel S, Werder EA, Schleiermacher E, Theile U, Wolff G, Wieacker P. Mutations of the androgen receptor gene in patients with complete androgen insensitivity. Hum Mutat 1997;9:57-61.

37. Hellwinkel OJ, Holterhus PM, Struve D, Marschke C, Homburg N, Hiort O. A unique exonic splicing mutation in the human androgen receptor gene indicates a physiologic relevance of regular androgen receptor transcript variants. J Clin Endocrinol Metab 2001;86:2569-2575.

38. Gast A, Neuschmid-Kaspar F, Klocker H, Cato AC. A single amino acid exchange abolishes dimerization of the androgen receptor and causes Reifenstein syndrome. Mol Cell Endocrinol 1995;111:93-98.

39. Ahmed SF, Cheng A, Dovey L, Hawkins JR, Martin H, Rowland J, Shimura $\mathrm{N}$, Tait AD, Hughes IA. Phenotypic features, androgen receptor binding, and mutational analysis in 278 clinical cases reported as androgen insensitivity syndrome. J Clin Endocrinol Metab 2000;85:658-665. 